\title{
Immunohistochemical Signatures as Predictors of Postsurgical Prognosis in Pulmonary Squamous Cell Carcinoma Patients
}

\section{Chengbin Lin}

Ningbo University Medical School

Hongyan Yu

Ningbo University Medical School

\section{Xiaohan Chen}

Ningbo University Medical School

\section{Weiyu Shen}

Ningbo Medical Treatment Centre Li Huili Hospital

Yong Xi ( $\nabla$ xiyong1211@163.com )

Ningbo Medical Treatment Centre Li Huili Hospital

\section{Research Article}

Keywords: Pulmonary squamous cell carcinoma, Immunohistochemical, Prognostic, Signature, Predictor

Posted Date: August 31st, 2021

DOl: https://doi.org/10.21203/rs.3.rs-829465/v1

License: (c) (i) This work is licensed under a Creative Commons Attribution 4.0 International License. Read Full License 


\section{Abstract}

\section{Background}

We aimed to evaluate the prognostic value of immunohistochemistry $(\mathrm{IHC})$ markers and tumor-nodemetastasis (TNM) stages in patients with pulmonary squamous cell carcinoma (SQCC).

\section{Methods}

From January 2010 to December 2014, aA total of 556 patients with SQCC who underwent radical resection were included. The patients were grouped into a discovery group $(n=334)$ and a validation group $(n=222)$. Using the least absolute shrinkage and selection operator regression model, we extracted IHCs that were associated with progression-free survival (PFS) and then built a classifier. Clinicopathologic variables and the IHC-based classifier were analysed using univariable and multivariable logistic regression analysis. A nomogram to predict PFS was constructed and validated using bootstrap resampling.

\section{Results}

FollowingUsing the least absolute shrinkage and selection operator regression model, four IHC markers associated with progression-free survival (PFS) were identified. Furthermore, we developed a nomogram integrating IHC markers to predict 3- and 5-year PFS. We used the IHC-based classifiers to stratify patients in both groups into high- and low-risk groups. PFS was better in the low-risk group than in the high-risk group in both the discovery and validation groups. Multivariate analysis demonstrated that the IHC-based classifiers were independently prognostic in predicting the PFS of patients with SQCC. The performance of the nomogram was evaluated and proven to be clinically useful.

\section{Conclusions}

We further developed a nomogram integrating the IHC-based classifiers and clinicopathological risk factors to predict PFS. Through combining the IHC-based classification and clinicopathology, we have a better insight into the prognosis assessment of patients with SQCC after surgery, which informs postoperative patient management.

\section{Introduction}

Lung cancer is one of the leading causes of death worldwide $(1,800,000$ deaths per year), with its incidence increasing every year (1)(1). Non-small cell lung cancer (NSCLC) is the most common type of lung cancer, accounting for $85 \%-90 \%$ of all lung cancer cases. The two major histologic types of lung cancers are squamous cell carcinoma and adenocarcinoma (2)(2). Radical resection is still the main treatment for squamous cell lung carcinoma_(3) (3). Although the tumor-node-metastasis (TNM) stage can predict the prognosis of patients, the prognosis for high-risk lung cancer patients is not hopeful (4) (4). Therefore, it is necessary.Fhis paper aims to improve patients' 3 yearpostoperative 
prognosis progression-free-survival (PFS) and 5-year PFS-by integrating multiple biomarker predictive models to guide patient management after surgery.

Microscopic morphological characteristics of tumors have always been the gold standard for lung cancer classification by the World Health Organization. In cases of poor tumor differentiation and in the absence of lung adenocarcinoma and cytological characteristics, classification is more difficult. Due to the need for precise tumor typing, reliance solely on morphology for diagnosis has been challenged in recent years. Owing to this, immunohistochemistry $(\mathrm{IHC})$ detection is important in the subtyping of NSCLC. IHC is widely used because it is simple, relatively inexpensive, and highly reliable. In clinical practice, in addition to $\mathrm{p} 63, \mathrm{p} 40$, and CK5/6, there are many epithelial cell markers, including 34BE12, desmocollin-3, S100A2, S100A7, SOX2, glypican 3, and miR-205, which have been used to identify squamous cell carcinoma (SQCC). A growing body of evidence suggests that IHC is a highly effective aid in predicting survival in patients with various cancer types. For example, High Ki67 has a close association with poor prognosis in colorectal cancer (5)(5), A three-gene IHC panel has been reported to predict the prognosis of patients with esophageal adenocarcinoma (6)(6). Several IHC-based biomarkers have been reported as predicting various cancers $(7)(7)$, but there are few reports pertaining to squamous cell carcinoma. The integration of multiple biomarkers into a single predictive model may enable clinicians to optimize patient treatment and reduce mortality.

The purpose of this study was to develop and validate IHC-based classifiers using the least absolute shrinkage and selection operator (LASSO) Cox regression model and to establish a prognosis for postoperative patients with pulmonary squamous cell carcinoma based on clinical-pathological parameters and IHC biomarkers.

\section{Methods}

\subsection{Patients and Samples}

Patients who underwent squamous cell carcinoma resection at Li Huili Hospital, affiliated with Ningbo University, from Januaryune $201 \underline{0} 4$ to Decemberdune $201 \underline{4} 9$ with complete clinical and immunological data were included $(N=556)$. The study was approved by the ethics committee of Li-h Huili Hospital and was conducted in accordance with the Helsinki Declaration. Informed consent was obtained from all patients prior to inclusion. Patients were randomly divided into the discovery group $(n=334)$ and the validation group $(n=222)$, a ratio of $6: 4$.

\section{$\underline{2.2}$ Immunohistochemistry}

The specimens studied were formalin-fixed and paraffin-enucleated tissue slices. Specimens were first analyzed by the primary pathologist then independently by two practicing pathologists. Specimens were then immunogrouped by the use of antibodies at the Ningbo Pathology Center. The antibodies used were from Fu Zhou Maixin Biotech and include CK pan, CK7, TTF-1, NapsinA, CK5/6, p63, p40, CD56, Syn, CgA, 
and Ki-67. All the specimens were fixed with $10 \%$ neutral formalin, embedded in paraffin, and stained with $3 \mu$-m section HE. Immunohistochemical staining was performed with EnVision.

\subsection{Development and Validation of IHC Markers}

The LASSO Cox regression model was used. Significant prognostic markers with non-zero coefficients were determined from the test group. The prognostic score for each patient was calculated using a linear combination of the identified markers. In the test group, a multimarker classifier to predict 3-and 5-year PFS in SQCC patients was established. The LASSO Cox regression model analysis was performed using the "glmnet" function in R software 3.0.1 (R Foundation for Statistical Computing, Vienna, Austria).

\subsection{Statistical Analysis}

We compared the two data sets using the $t$-test for continuous variables and the chi-square test for categorical variables. The Kaplan-Meier survival analysis and log-rank were used to estimate the survival time of patients in the different risk groups stratified according to $\mathrm{IHC}$ markers. A quantile plot was used to select the best cut-off point for patient survival time.

ROC curve analysis was performed, statistically significant prognostic markers were analyzed using univariate and multivariate Cox regressions, and Cox regression coefficients were used to build a column chart to predict PFS probability. Based on the regression analysis, a calibration plot was obtained. We evaluated the clinical utility of the charts through decision curve analysis (DCA). R's "rms" function was used for line and calibrations plots. The two-tailed $p$-value was 0.05 .

\section{Results}

\subsection{Patients' Clinical Characteristics}

All patients had undergone SQCC resection. The clinical stage of patients was determined according to the TNM staging of lung cancer in the 8th edition of the UICC (8)(8). Detailed clinical-pathological characteristics of the discovery group $(n=334)$ and the validation group $(n=222)$ are in Table 1 .

Table 1. Pathoclinical characteristics of patients in discovery and validation cohort. 


\begin{tabular}{|c|c|c|c|c|}
\hline- & $\begin{array}{l}\text { Train Low } \\
\text { risk }\end{array}$ & $\begin{array}{l}\text { Train High } \\
\text { risk }\end{array}$ & $\frac{\text { Test Low }}{\text { risk }}$ & $\frac{\text { Test High }}{\text { risk }}$ \\
\hline - & $(\underline{\mathrm{N}=132})$. & $(\underline{\mathrm{N}=202})$. & $(\mathrm{N}=90)$. & $(\mathrm{N}=132)$. \\
\hline Age & - & - & - & - \\
\hline$\leqq 60$ & $43(\underline{32.6 \%})$ & $66(\underline{32.7 \%})$. & 31 (34.4\%). & $46(\underline{34.8 \%})$. \\
\hline$>60$ & $\underline{89}(67.4 \%)$. & $136(67.3 \%)$ & $\underline{59}(\underline{65.6 \%})$. & $\underline{86}(\underline{65.2 \%})$. \\
\hline Smoke & - & - & - & - \\
\hline$\underline{N}$ & $47(\underline{35.6 \%})$ & $\underline{57}(\underline{28.2 \%})$. & $\underline{38}(\underline{42.2 \%})$. & $\underline{42}(\underline{31.8 \%})$ \\
\hline$\underline{Y}$ & $\underline{85}(64.4 \%)$. & $\underline{145}(\underline{71.8 \%})$ & $\underline{52}(\underline{57.8 \%})$. & $\underline{90}(\underline{68.2 \%})$. \\
\hline Gender & - & - & - & - \\
\hline F & $4 \underline{(3.0 \%)}$. & $9(4.5 \%)$. & $\underline{7}(\underline{7.8 \%})$. & $10(\underline{7.6 \%})$. \\
\hline$\underline{M}$ & $\underline{128}(\underline{97.0 \%})$ & $193(\underline{95.5 \%})$ & 83 $(\underline{92.2 \%})$ & 122 (92.4\%). \\
\hline Iymphatic.or.blood.vessel.invasion & - & - & - & - \\
\hline$\underline{N}$ & $\underline{110}(\underline{83.3 \%})$. & $\underline{157}(\underline{77.7 \%})$. & $\underline{76}(\underline{84.4 \%})$ & $\underline{107}(\underline{81.1 \%})$. \\
\hline$\underline{Y}$ & $\underline{22}(\underline{16.7 \%})$ & $\underline{45}(\underline{22.3 \%})$ & $\underline{14}(\underline{15.6 \%})$. & $\underline{25}(\underline{18.9 \%})$ \\
\hline Nerve.invasion & - & - & - & - \\
\hline$\underline{N}$ & $\underline{115}(\underline{87.1 \%})$ & $\underline{174}(\underline{86.1 \%})$. & $\underline{82}(\underline{91.1 \%})$ & $\underline{121}(\underline{91.7 \%})$. \\
\hline$\underline{Y}$ & $\underline{17}(\underline{12.9 \%})$. & $\underline{28}(\underline{13.9 \%})$ & $\underline{8}(\underline{8.9 \%})$. & $\underline{11}(\underline{8.3 \%})$. \\
\hline Pleural.invasion. & - & - & - & - \\
\hline$\underline{N}$ & $\underline{118}(\underline{89.4 \%})$ & $\underline{159}(\underline{78.7 \%})$. & $\underline{86}(\underline{95.6 \%})$ & $\underline{115}(\underline{87.1 \%})$ \\
\hline$\underline{Y}$ & $\underline{14}(\underline{10.6 \%})$. & $\underline{43}(\underline{21.3 \%})$ & $\underline{4}(\underline{4.4 \%})$ & $17(\underline{12.9 \%})$. \\
\hline Differention & - & - & - & - \\
\hline$\underline{\mathrm{w}}$ & $\underline{21}(\underline{15.9 \%})$ & $\underline{22}(\underline{10.9 \%})$ & $10(\underline{11.1 \%})$. & $\underline{13}(\underline{9.8 \%})$. \\
\hline$\underline{L}$ & $\underline{47}(\underline{35.6 \%})$ & $\underline{82}(\underline{40.6 \%})$. & $\underline{34}(\underline{37.8 \%})$. & $\underline{50}(\underline{37.9 \%})$ \\
\hline$\underline{M}$ & $\underline{64}(\underline{48.5 \%})$ & $\underline{98}(\underline{48.5 \%})$ & $\underline{46}(\underline{51.1 \%})$ & $\underline{69}(\underline{52.3 \%})$ \\
\hline Stage.1 & - & - & - & - \\
\hline$\underline{1}$ & $\underline{76}(\underline{57.6 \%})$ & $\underline{64}(\underline{31.7 \%})$ & $\underline{55}(\underline{61.1 \%})$ & $\underline{55}(\underline{41.7 \%})$ \\
\hline$\underline{11}$ & $\underline{36}(\underline{27.3 \%})$ & $\underline{55}(\underline{27.2 \%})$ & $\underline{21}(\underline{23.3 \%})$ & $\underline{33}(\underline{25.0 \%})$ \\
\hline III & $\underline{20}(15.2 \%)$ & $\underline{83}(\underline{41.1 \%})$ & $\underline{14}(\underline{15.6 \%})$ & $\underline{44}(\underline{33.3 \%})$ \\
\hline
\end{tabular}




\subsection{Selection and Grouping of IHC Markers}

The LASSO Cox regression model was used to identify potential predictive IHC markers (SOX2, CEA, Ki-67, p53, CK5/6, 34BE12, p63, CD44V6, COX-2, PGP-9.5, EGFR, VEGF, c-Myc, BCL-2, Topo-II, cyclinD1, p16, p21, p27; Fable-Fig.1Z). We used the LASSO method to reduce the regression coefficient and reduce 19 characteristics to 4 potential predictors: SOX2, VEGF, p63, and p27. Risk score $=0.063372939 \times$ SOX2 $0.004970944 \times \mathrm{p} 63+0.106611323 \times$ VEGF $-0.013048563 \times \mathrm{P} 27$. In this formula, a negative status of $\mathrm{IHC}$ is expressed as 0 and a positive status as 1 . Consequently, patients with scores greater than or equal to 0 ( $n=202,60.5 \%)$ were classified as high risk; those with scores less than $0(n=132,39.5 \%)$ were classified as low risk(Fig.2). PFS was significantly better in the low-risk group.

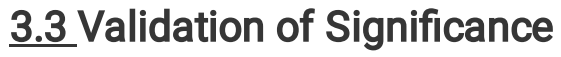

There were significant differences in survival rates among patients in different risk groups. PFS was significantly better in the low-risk subgroups than in the high-risk subgroups in the discovery group, the validation group, and the cohort as a whole. The same analysis was carried out in the validation group ( $n$ $=222)$. Using the risk score, we classified these patients into a high-risk group $(n=132,59.5 \%)$ and a lowrisk group ( $n=90,40.5 \%$ ). The five-yearPFS was ( for the high-risk group and () for the low-risk group.Similar differences between the two groups were noted in the combined discovery and validation cohort (Fig. $\underline{3} Z)$.

\subsection{Prediction Accuracy}

Pleural invasion, staging, and $\mathrm{IHC}$ markers were significant prognostic factors in the univariate analysis. There was no statistically significant difference in other clinical-pathological factors. The multivariate analysis identified tumor-stage markers and IHC markers to be independent PFS predictors. Furthermore, combining IHC-based classifiers with staging provided better predictive value than $\mathrm{IHC}$-based classifiers or staging alone. Therefore, $\mathrm{IHC}$-based classifiers can increase the prognostic value of staging in postresection SQCC patients (Fig. $\underline{4} 3$ and Fig. $\underline{5} 4$ ).

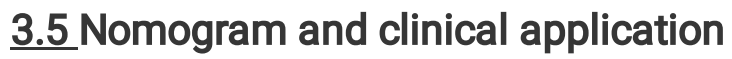

To provide a clinically relevant tool for predicting prognosis, we built a nomogram that integrates a variety of clinical-pathological risk factors based on IHC markers and PFS. Predictive factors include pleural invasion, tumor stage, and IHC markers. The calibration curve showed optimal performance of the nomogram with a high degree of consistency between the PFS and the Kaplan-Meier estimates. (Fig. $\underline{6} 5$ ):

DCA was used to evaluate the clinical utility of a nomogram based on IHC markers by quantifying net benefits. The threshold probability of a patient choosing treatment tells us how the patient weighs the relative harm of false positive and false negative predictions. Here, the relative harm from treatment is equal to that of avoiding the expected benefits of treatment. Net benefit was calculated by subtracting the proportion of false positives from the sum of false and true positives. The nomogram proved to be of 
clinical value as it ensured a better net benefit in comparison to full or no treatment options-.. $\mathrm{Fig} . \underline{-7}$ and Fig.

\section{Discussion}

Surgical resection is the most effective treatment for lung cancer, but predicting postoperative patients' likelihood of recurrence or PFS is difficult (9)(9). The current method does not provide viable clinical prognostic markers in postoperative patients with squamous cell lung carcinoma (10)(10). To help guide decision-making in the management of SQCC patients, we used a group of SQCC patients to analyze IHC markers that were associated with prognosis and developed a nomogram to estimate 3- and 5-year PFS in postoperative patients.

A LASSO Cox regression was used to identify statistically significant prognostic factors; this regression builds models based on both predictors and selected characteristics. In a recent study, the LASSO Cox regression method was used in multivariate analysis panels such as preoperative prediction of lymph node metastasis in colorectal cancer (11)(11). Our study used the LASSO method to reduce the regression coefficient and reduce 19 characteristics to 4 potential predictors. Patients were divided into low-risk and high-risk groups by incorporating $4 \mathrm{IHC}$ markers into the classifier. The survival rate of patients in the low-risk group was significantly higher than that in the high-risk group. In addition, we validated the potential value of $\mathrm{IHC}$ markers in predicting patient prognosis using the validation group. Multivariate analysis showed that IHC markers are independent prognostic factors of PFS after adjustment is made for clinical-pathological variables. Combined with staging, IHC markers provide better predictive value than staging alone. $\mathrm{IHC}$ and clinical-pathological variables including invasion status and staging were included in the nomogram. The calibration graph showed significant correlation between the predicted survival probability and the observed survival rate. DCA showed great potential for clinical applicability of the nomogram.

IHC technology has advanced from traditional cell microscopic examination technology. It provides a better understanding of enzyme activity and the shape and structure of the patient's cells, as well as a more accurate understanding of the histopathology of different lesions (12)(12). It is highly accurate and specific. SOX2 is an important member of the SOX family, which regulates the development of embryos and tissues by binding to the HMG domain of the target gene, and it maintains the pluripotent, undifferentiated state of the stem cells, regulates their proliferation, and determines their cell fate (13) (13). Numerous studies have found SOX2 expression abnormalities in many solid tumors, such as breast cancer and malignant gliomas (14)(14). One study has shown that amplification of SOX2 loci can be detected in non-small cell lung cancer, which is associated with median overall survival in early patients with the disease but not in patients with advanced tumors (15)(15). Another study showed that the prognosis of tumor patients with a high expression of SOX2 was significantly better than that of patients with a low expression (16)(16). Interestingly, Chou and his colleagues reported that the expression of SOX2 was associated with the staging of NSCLC (17)(17). As for lymph node metastasis and TNM stage, the lower the degree of tumor differentiation, the earlier the metastasis to the lymph nodes; the later the 
TNM stage, the higher the expression rate of SOX2 (18)(18). This suggests that the expression of SOX2 may be closely related to the proliferation and metastasis process of NSCLC and a relevant factor in poor tumor prognosis. Therefore, it is proposed that SOX2 expression can be used to predict NSCLC.

The human p63 gene, located on chromosomes 3q27-3q29, consists of 15 exons that each consist of two independent initiators. At present, the p63 (monoclonal antibody cloning system 4A4) used in domestic and foreign pathology laboratories can identify TAp63 and xenon Np63, and it is considered to be a "broad-spectrum" p63 antibody, while the p40 antibody Np63 only recognizes p-Np63 (19)(19). The protein p63 is often expressed at the base of the epithelial tissue, playing an important role in the formation of normal endothelial tissue, and it is expressed in a variety of malignant tumors of squamous cell origin, especially in squamous cell carcinoma tissue. Its frequency and expression distribution are related to the degree of SQCC differentiation (20)(20). In breast cancer, the expression of p63 is demonstrated to be related to tumor grading and is significant for the prognosis and treatment index of tumors (21)(21).

The protein p27Kip1 is a cell cycle inhibitor with a relative molecular mass of 27,000 discovered in a 1994 study of cell suppression mechanisms. Studies have found that p27Kip1 is reduced or missing in a variety of malignant tumor tissues, including esophageal, colon, prostate, bladder, liver, breast, and nonsmall cell lung cancer (22-28)(22 28). The expression of p27 as identified via RT-PCR was significantly lower than that in adjacent paracancerous tissues in 230 cases of NSCLC. There were significant differences $(p<0.050)$ in lymph node metastasis, TNM staging, and differentiation classification (29) (29).

VEGF is the most consequential cytokine known to promote the growth of the endothelium of blood vessels. It can increase vascular permeability, promote tumor vascular formation, and play an important role in the proliferation and migration of tumor vascular endothelial cells. In vitro animal model experiments have shown that inhibition of the expression of VEGF in tumors can significantly inhibit the formation of blood vessels in lung cancer cell tissue, reduce tumor volume, and improve the survival time in mice (28)[28](30). Other studies have shown that VEGF increased significantly in NSCLC patients, and its elevation was associated with tumor TNM stage and lymph node metastasis (30)(37). A metaanalysis suggested that VEGF increases the risk of lung cancer deterioration, that VEGF may be involved in the leaching metastasis of non-small cell lung cancer, and it proposes that VEGF accelerates the malignant progression of tumors (31)_(32).

In this study, we used the LASSO Cox regression model to successfully integrate multiple IHC markers into a singular model that had higher predictive power than a single IHC marker. Although IHC markerbased nomograms can predict the survival of patients with lung squamous cancer highly accurately, there are some limitations to our study. First, the nomogram is based on retrospective data from a single center. Second, our study lacks the data on the genetic characteristics needed to validate the biomarkers. Third, further prospective studies of multicenter clinical trials are needed to further validate the results. In summary, we have developed and validated a nomogram combining IHC markers and clinical- 
pathological characteristics to accurately predict the prognosis of patients with squamous cell lung carcinoma. An accurate prognostic model for predicting patient survival can be of significant value in choosing the best treatment strategy and individualizing patient care.

\section{Abbreviations}

IHC: immunohistochemistry

TNM: tumor-node-metastasis

SQCC: pulmonary squamous cell carcinoma

PFS: progression-free survival

NSCLC: Non-small cell lung cancer

LASSO: least absolute shrinkage and selection operator

DCA: decision curve analysis

\section{Declarations}

\section{knowledgements}

Thanks are due to the Ningbo Pathology Center for assistance with the study.

\section{Funding}

This work was supported by the major science and technology innovation in 2025 projects of Ning $\underline{\text { bo, }}$ China (Grant No.2019B10039).

\section{Authors' contributions}

Chengbin Lin, Weiyu Shen, Yong Xi participated in the study design, and Hongyan Yu, Xiaohan Chen statistically analyzed the data. The manuscript was drafted by Chengbin Lin and revised by Yong Xi and Feng Weiyu Shen. The authors read and approved the final manuscript.

\section{Availability of data and materials}

The datasets supporting the conclusion of this article are included within the article.

\section{Declarations}

\section{Ethics approval and consent to participate}

Not applicable 


\section{Consent for publication}

Not applicable

\section{Competing interests}

The authors declare that they have no competing interests.

\section{References}

1. Sung H, Ferlay J, Siegel RL, Laversanne M, Soerjomataram I, Jemal A, et al. Global Cancer Statistics 2020: GLOBOCAN Estimates of Incidence and Mortality Worldwide for 36 Cancers in 185 Countries. CA: a cancer journal for clinicians. 2021;71(3):209-49.

2. Youlden DR, Cramb SM, Baade PD. The International Epidemiology of Lung Cancer: geographical distribution and secular trends. Journal of thoracic oncology : official publication of the International Association for the Study of Lung Cancer. 2008;3(8):819-31.

3. Datta D, Lahiri B. Preoperative evaluation of patients undergoing lung resection surgery. Chest. 2003;123(6):2096-103.

4. Goldstraw P, Crowley J, Chansky K, Giroux DJ, Groome PA, Rami-Porta R, et al. The IASLC Lung Cancer Staging Project: proposals for the revision of the TNM stage groupings in the forthcoming (seventh) edition of the TNM Classification of malignant tumours. Journal of thoracic oncology : official publication of the International Association for the Study of Lung Cancer. 2007;2(8):706-14.

5. Tong G, Zhang G, Liu J, Zheng Z, Chen Y, Niu P, et al. Cutoff of 25\% for Ki67 expression is a good classification tool for prognosis in colorectal cancer in the AJCC-8 stratification. Oncology reports. 2020;43(4):1187-98.

6. Ong CA, Shapiro J, Nason KS, Davison JM, Liu X, Ross-Innes C, et al. Three-gene immunohistochemical panel adds to clinical staging algorithms to predict prognosis for patients with esophageal adenocarcinoma. Journal of clinical oncology : official journal of the American Society of Clinical Oncology. 2013;31(12):1576-82.

7. Cagle PT, Allen TC, Olsen RJ. Lung cancer biomarkers: present status and future developments. Archives of pathology \& laboratory medicine. 2013;137(9):1191-8.

8. Chansky K, Detterbeck FC, Nicholson AG, Rusch VW, Vallières E, Groome P, et al. The IASLC Lung Cancer Staging Project: External Validation of the Revision of the TNM Stage Groupings in the Eighth Edition of the TNM Classification of Lung Cancer. Journal of thoracic oncology : official publication of the International Association for the Study of Lung Cancer. 2017;12(7):1109-21. 
9. Taylor MD, Nagji AS, Bhamidipati CM, Theodosakis N, Kozower BD, Lau CL, et al. Tumor recurrence after complete resection for non-small cell lung cancer. The Annals of thoracic surgery. 2012;93(6):1813-20; discussion 20-1.

10. Xi JJ, Yin JC, Wang L, Lu CL, Wang Q, Jiang W. A surveillance method-oriented detection of postoperative spatial-temporal recurrence for non-small cell lung cancer. Journal of thoracic disease. 2018;10(11):6107-17.

11. Huang YQ, Liang CH, He L, Tian J, Liang CS, Chen X, et al. Development and Validation of a Radiomics Nomogram for Preoperative Prediction of Lymph Node Metastasis in Colorectal Cancer. Journal of clinical oncology : official journal of the American Society of Clinical Oncology. 2016;34(18):2157-64.

12. Jain D, Nambirajan A, Borczuk A, Chen G, Minami Y, Moreira AL, et al. Immunocytochemistry for predictive biomarker testing in lung cancer cytology. Cancer cytopathology. 2019;127(5):325-39.

13. Novak D, Hüser L, Elton JJ, Umansky V, Altevogt P, Utikal J. SOX2 in development and cancer biology. Seminars in cancer biology. 2020;67(Pt 1):74-82.

14. Gangemi RM, Griffero F, Marubbi D, Perera M, Capra MC, Malatesta P, et al. SOX2 silencing in glioblastoma tumor-initiating cells causes stop of proliferation and loss of tumorigenicity. Stem cells (Dayton, Ohio). 2009;27(1):40-8.

15. Toschi L, Finocchiaro G, Nguyen TT, Skokan MC, Giordano L, Gianoncelli L, et al. Increased SOX2 gene copy number is associated with FGFR1 and PIK3CA gene gain in non-small cell lung cancer and predicts improved survival in early stage disease. PloS one. 2014;9(4):e95303.

16. Ying J, Shi C, Li CS, Hu LP, Zhang WD. Expression and significance of SOX2 in non-small cell lung carcinoma. Oncology letters. 2016;12(5):3195-8.

17. Chou YT, Lee CC, Hsiao SH, Lin SE, Lin SC, Chung CH, et al. The emerging role of SOX2 in cell proliferation and survival and its crosstalk with oncogenic signaling in lung cancer. Stem cells (Dayton, Ohio). 2013;31(12):2607-19.

18. lijima Y, Seike M, Noro R, Ibi T, Takeuchi S, Mikami I, et al. Prognostic significance of PIK3CA and SOX2 in Asian patients with lung squamous cell carcinoma. International journal of oncology. 2015;46(2):505-12.

19. Bishop JA, Teruya-Feldstein J, Westra WH, Pelosi G, Travis WD, Rekhtman N. p40 $(\Delta N p 63)$ is superior to $\mathrm{p} 63$ for the diagnosis of pulmonary squamous cell carcinoma. Modern pathology : an official journal of the United States and Canadian Academy of Pathology, Inc. 2012;25(3):405-15.

20. Saghravanian N, Anvari K, Ghazi N, Memar B, Shahsavari M, Aghaee MA. Expression of p63 and CD44 in oral squamous cell carcinoma and correlation with clinicopathological parameters. Archives of 
oral biology. 2017;82:160-5.

21. Constantinou C, Papadopoulos S, Karyda E, Alexopoulos A, Agnanti N, Batistatou A, et al. Expression and Clinical Significance of Claudin-7, PDL-1, PTEN, c-Kit, c-Met, c-Myc, ALK, CK5/6, CK17, p53, EGFR, Ki67, p63 in Triple-negative Breast Cancer-A Single Centre Prospective Observational Study. In vivo (Athens, Greece). 2018;32(2):303-11.

22. Shibata $\mathrm{H}$, Matsubara $\mathrm{O}$, Wakiyama $\mathrm{H}$, Tanaka S. The role of cyclin-dependent kinase inhibitor p27 in squamous cell carcinoma of the esophagus. Pathology, research and practice. 2001;197(3):15764.

23. Dai JY, Liang XP, Wen JL, Li CY, Deng CZ, Zhang ZH. [Expression of P27 protein and cyclin E in colon cancer]. Ai zheng $=$ Aizheng $=$ Chinese journal of cancer. 2003;22(10):1093-5.

24. Ribal MJ, Fernandez PL, Lopez-Guillermo A, Farré X, Santos Y, Gibanel R, et al. Low p27 expression predicts biochemical relapse after radical prostatectomy in patients with clinically localised prostate cancer. Anticancer research. 2003;23(6d):5101-6.

25. Lacoste-Collin L, Gomez-Brouchet A, Escourrou G, Delisle MB, Levade T, Uro-Coste E. Expression of p27(Kip1) in bladder cancers: immunohistochemical study and prognostic value in a series of 95 cases. Cancer letters. 2002;186(1):115-20.

26. Lei PP, Zhang ZJ, Shen LJ, Li JY, Zou Q, Zhang HX. Expression and hypermethylation of p27 kip1 in hepatocarcinogenesis. World journal of gastroenterology. 2005;11(29):4587-91.

27. Sgambato A, Zhang YJ, Arber N, Hibshoosh H, Doki Y, Ciaparrone M, et al. Deregulated expression of p27(Kip1) in human breast cancers. Clinical cancer research : an official journal of the American Association for Cancer Research. 1997;3(10):1879-87.

28. Huang H, Salavaggione O, Rivera L, Mukherjee S, Brekken R, Tennant B, et al. Woodchuck VEGF (wVEGF) characteristics: Model for angiogenesis and human hepatocellular carcinoma directed therapies. Archives of biochemistry and biophysics. 2019;661:97-106.

29. Yang L, Yang J. Expression and clinical significance of microRNA-21, PTEN and p27 in cancer tissues of patients with non-small cell lung cancer. Oncology letters. 2020;20(4):49.

30. Zheng CL, Qiu C, Shen MX, Qu X, Zhang TH, Zhang JH, et al. Prognostic impact of elevation of vascular endothelial growth factor family expression in patients with non-small cell lung cancer: an updated meta-analysis. Asian Pacific journal of cancer prevention : APJCP. 2015;16(5):1881-95.

31. Fan J, Zhang W, Lei C, Qiao B, Liu Q, Chen Q, et al. Vascular endothelial growth factor polymorphisms and lung cancer risk. International journal of clinical and experimental medicine. 2015;8(4):6406-11. 


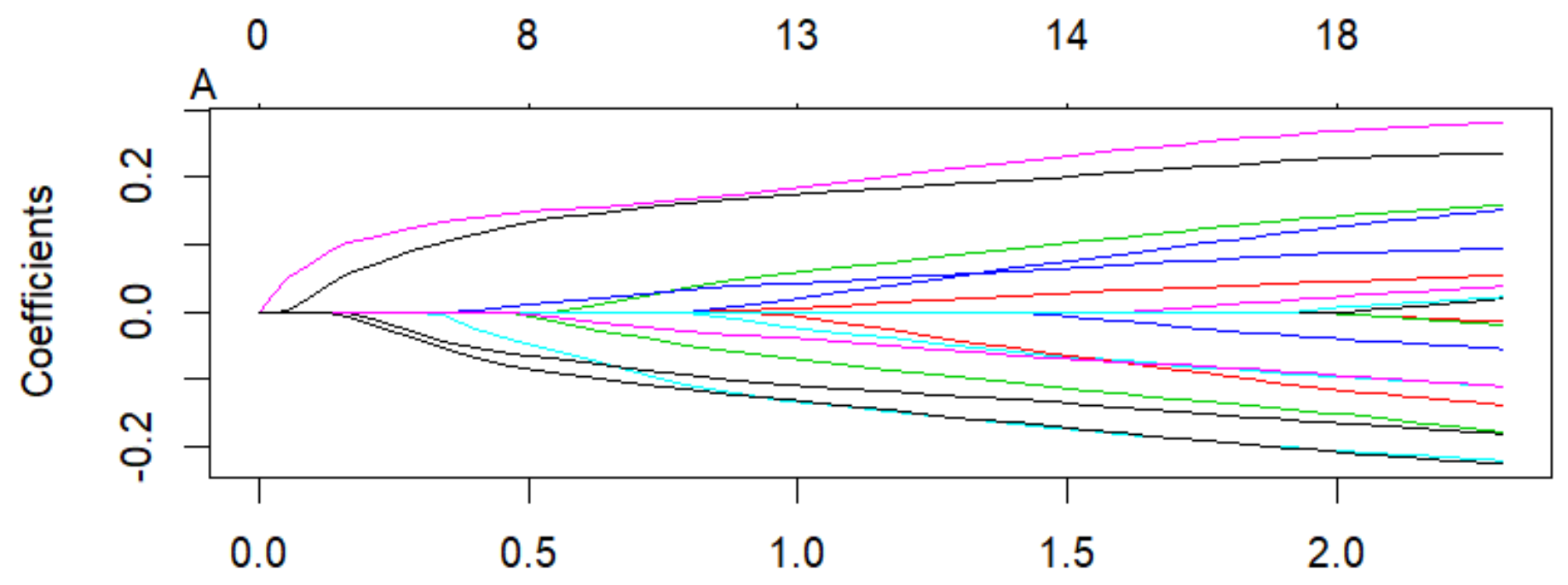

\section{L1 Norm}

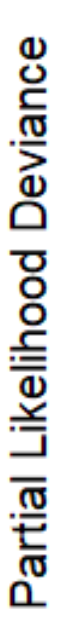

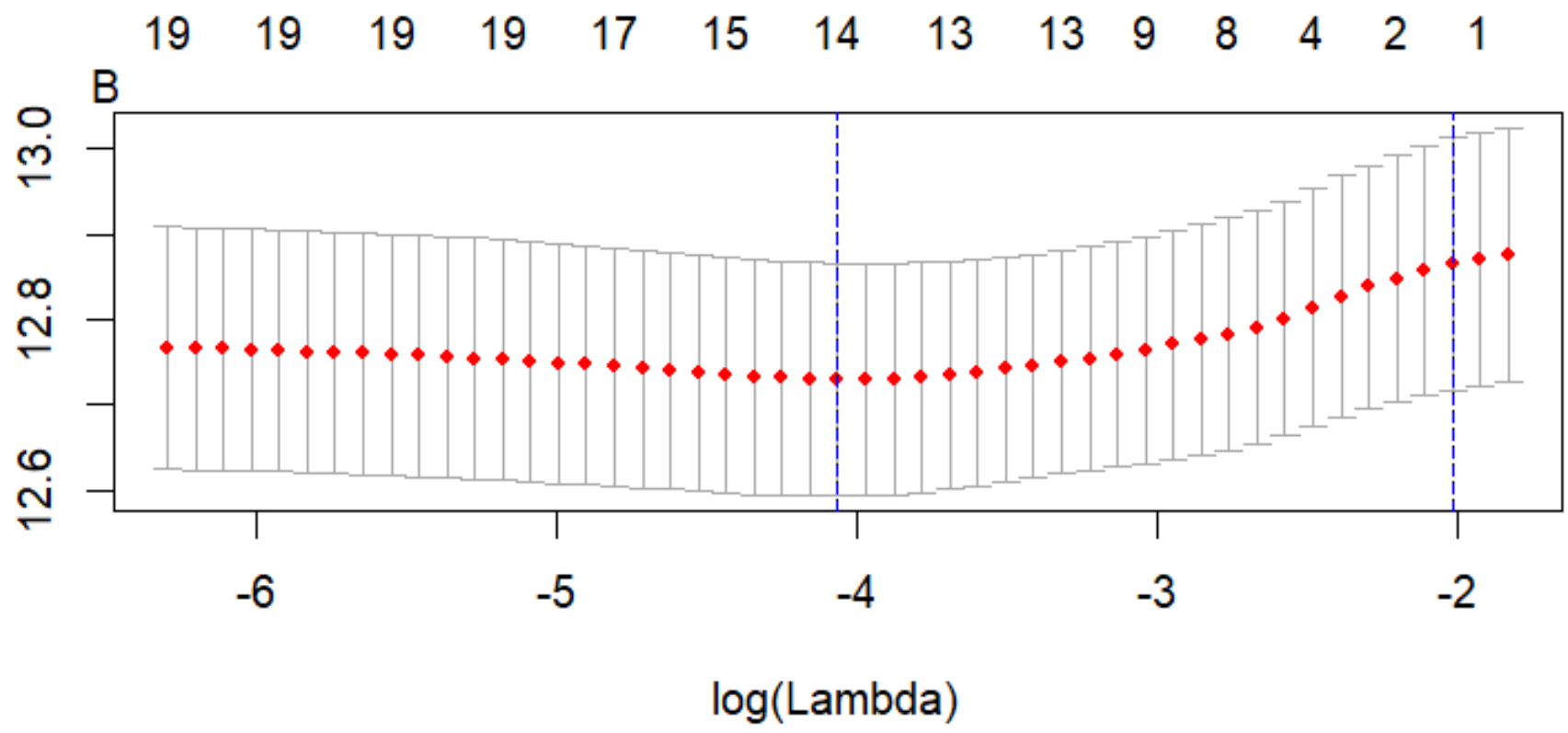

\section{Figure 1}

Feature selection using LASSO regression model. (A) Tuning parameter (selection by 10 -fold crossvalidation via minimum criteria). Partial likelihood deviance was plotted versus log(c). (B) Coefficient profile of the IHC markers associated with PFS of patients with SQCC. The vertical line is shown at the optimal value with four nonzero coefficients. 

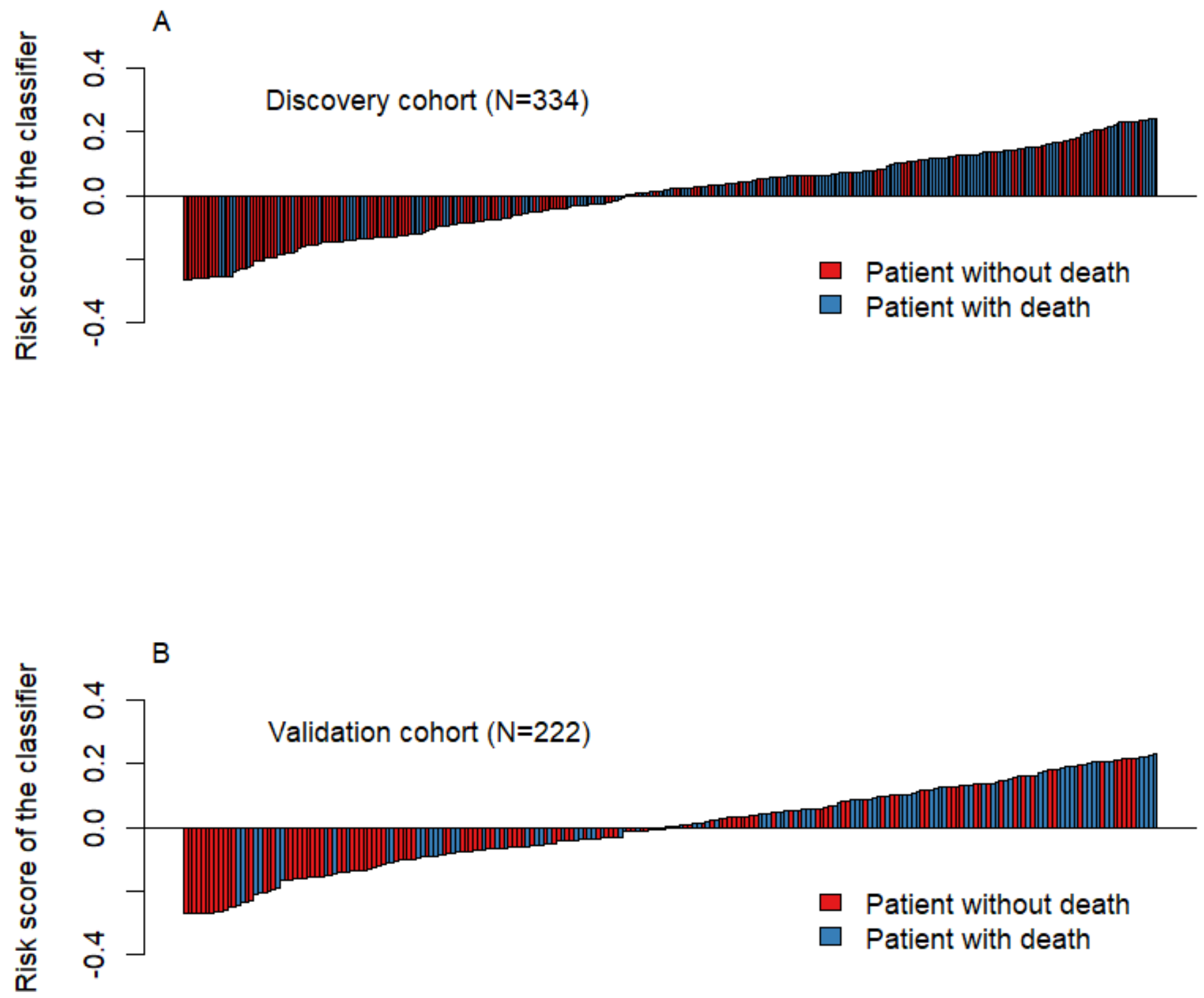

Figure 2

Distribution of risk score by 4-IHC-based classifier. (A) Discovery cohort and (B) validation cohort. 

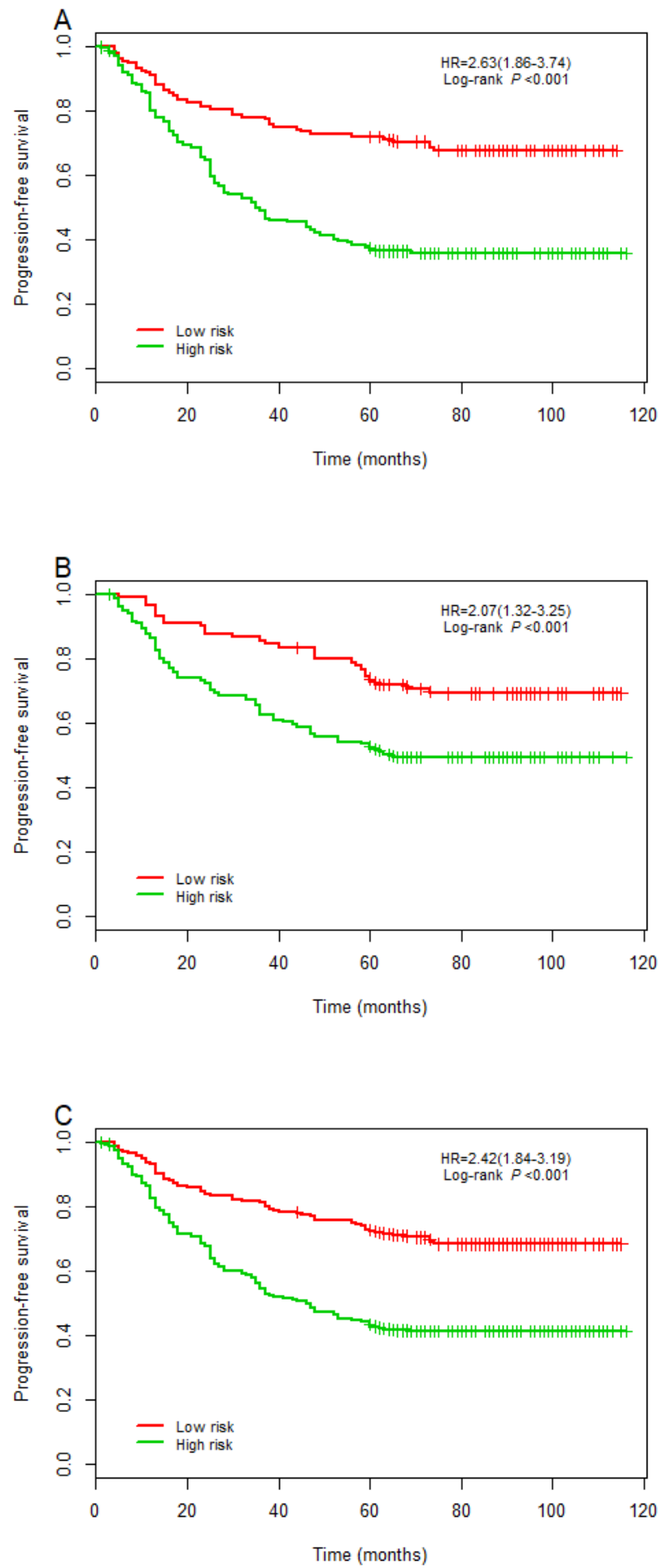

Figure 3

The PFS in high-risk group and low-risk group stratified by IHC signature. (A) Discovery cohort, (B) validation cohort, and (C) the combined cohort of discovery and validation groups. 
Age (>60 vs. $\leq 60)$

Smoke (Yes vs. No)

Gender (Male vs. Female)

lymphatic or blood vessel invasion (Yes vs. No)

Nerve invasion (Yes vs. No)

Pleural invasion (Yes vs. No)

Differention ( $L$ vs. W)

Differention (M vs. W)

Stage (II vs. I)

Stage (III vs. I)

Based classifier

Pleural invasion (Yes vs. No)

Stage (II vs. I)

Stage (III vs. I)

Based classifier

$P$
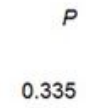

0.102

0.537

0.004

0.128

0.000

0.081

0.204

0.000

0.000

0.000
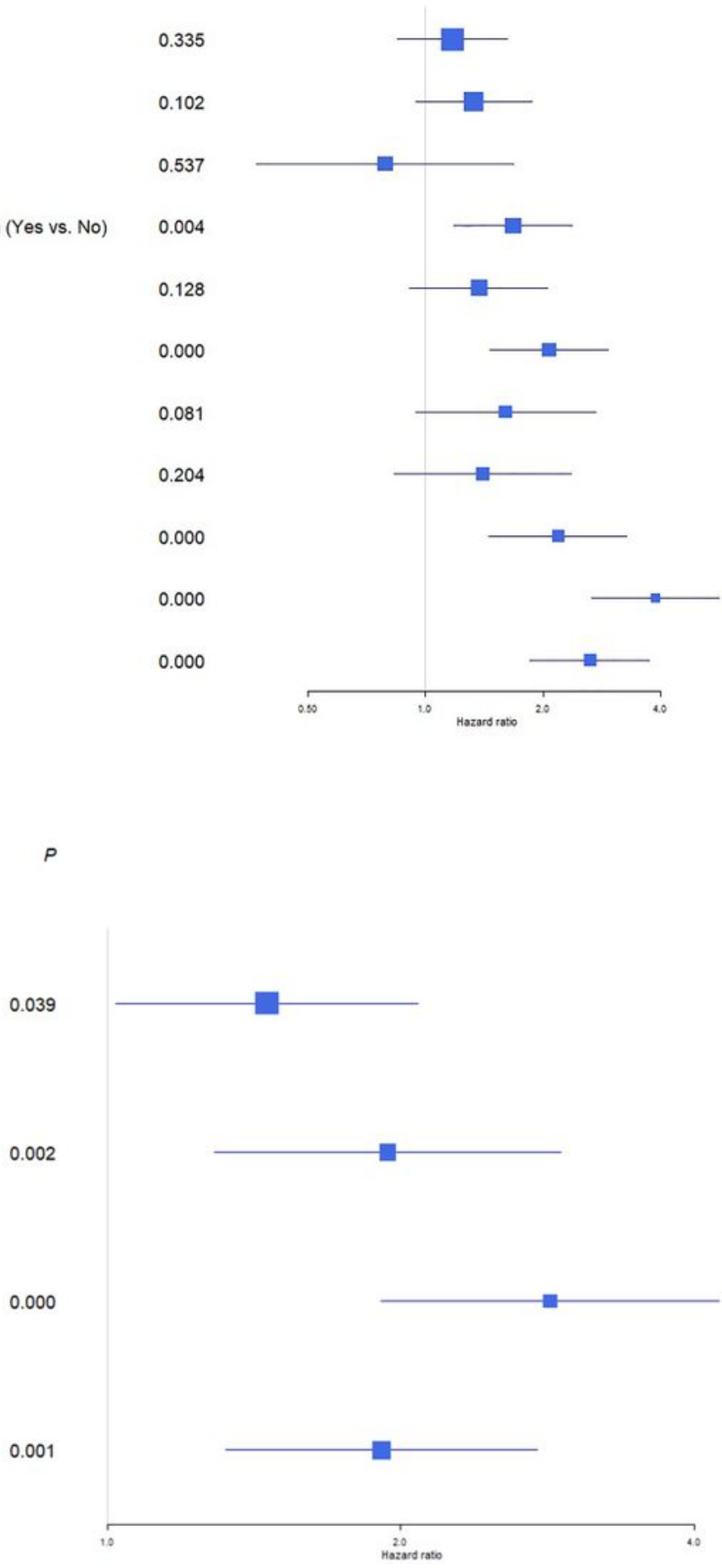

\section{Figure 4}

Analysis of clinicopathological information with PFS. (A) Univariate and (B) multivariate analysis of clinicopathological information with PFS 


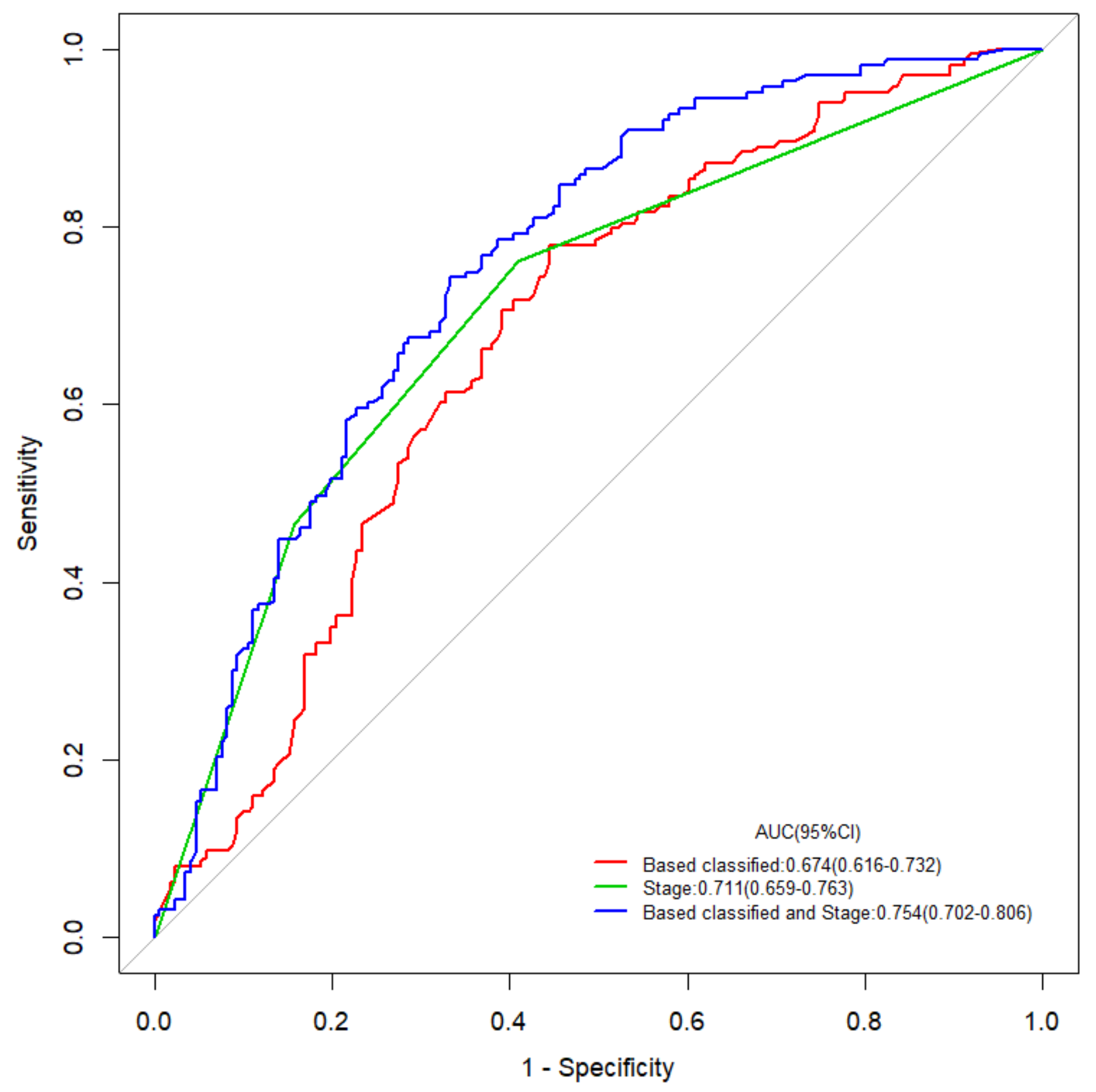

Figure 5

ROC curve analysis compares the prognostic value of IHC signature with tumor stage. 
Points

$\begin{array}{llllllllll}0 & 10 & 20 & 30 & 40 & 50 & 60 & 70 & 80 & 90\end{array}$

Pleural invasion

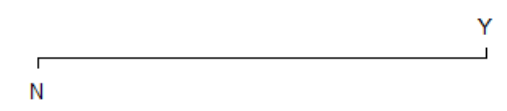

Stage

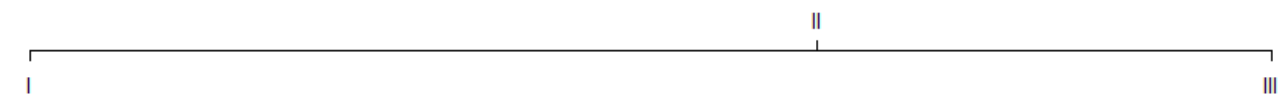

Based classified

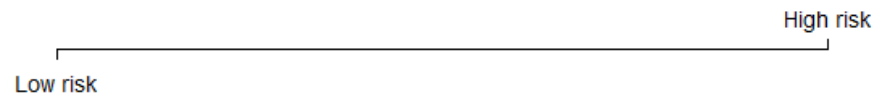

Total Points

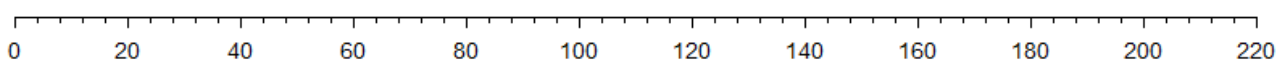

3-year PFS

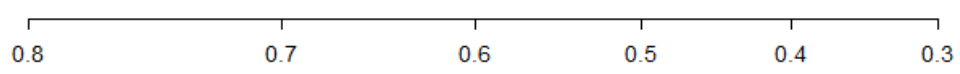

5-year PFS

\begin{tabular}{llllll}
\hline 0.7 & 1 & 1 & 1 & \\
0.6 & 0.5 & 0.4 & 0.3 & 0.2
\end{tabular}

Figure 6

Nomogram integrating IHC markers and clinicopathological factors. 


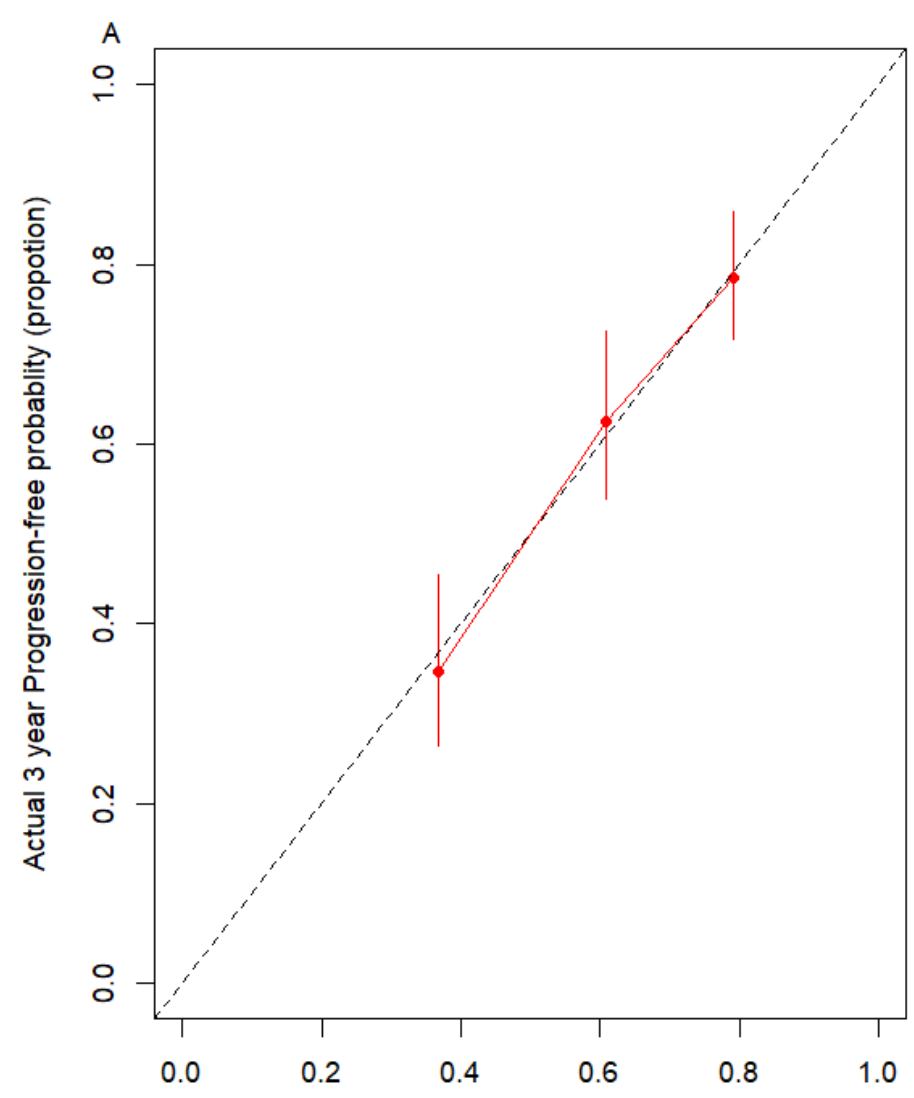

Predicted probability of 3 year Progression-free probablity

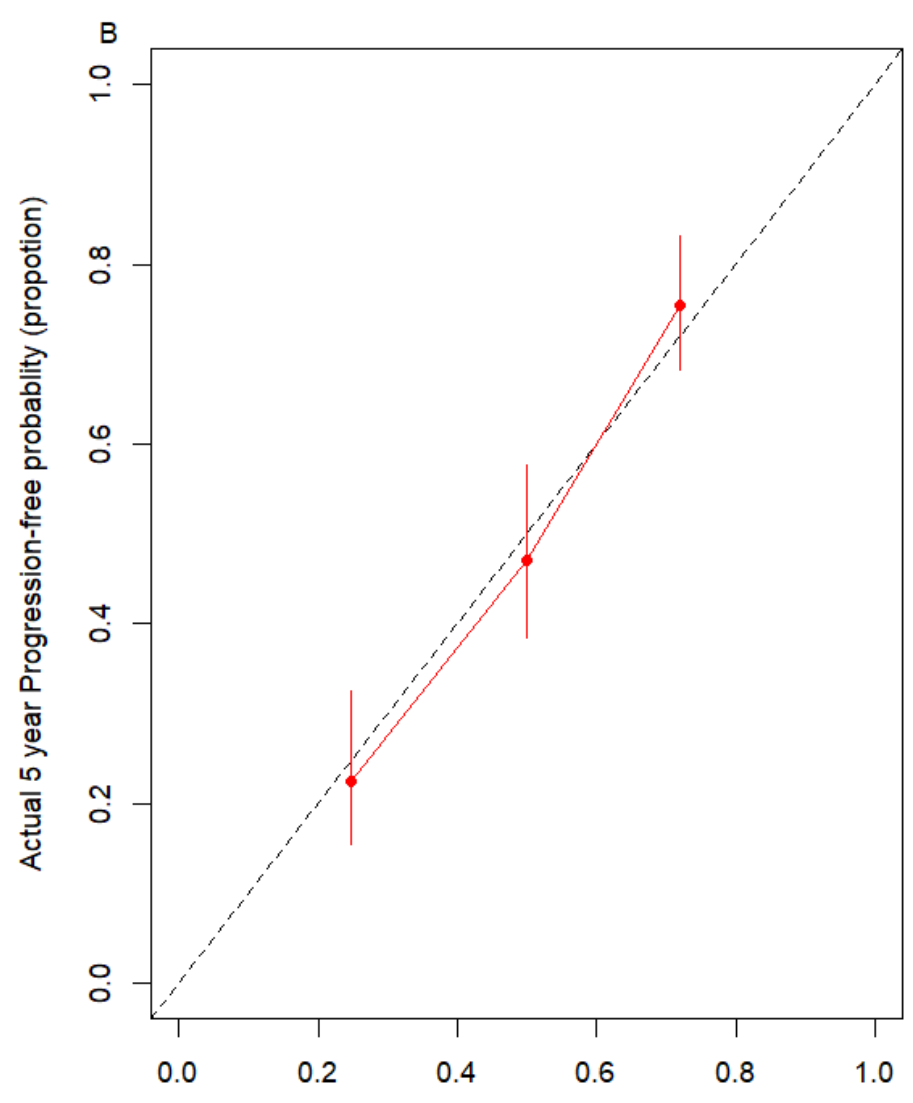

Predicted probability of 5 year Progression-free probablity

Figure 7

Evaluation of nomogram using calibration curves: 3- and 5-year nomogram calibration curves. The dashed line represents an ideal evaluation, whereas the red line represents the performance of the nomogram. 

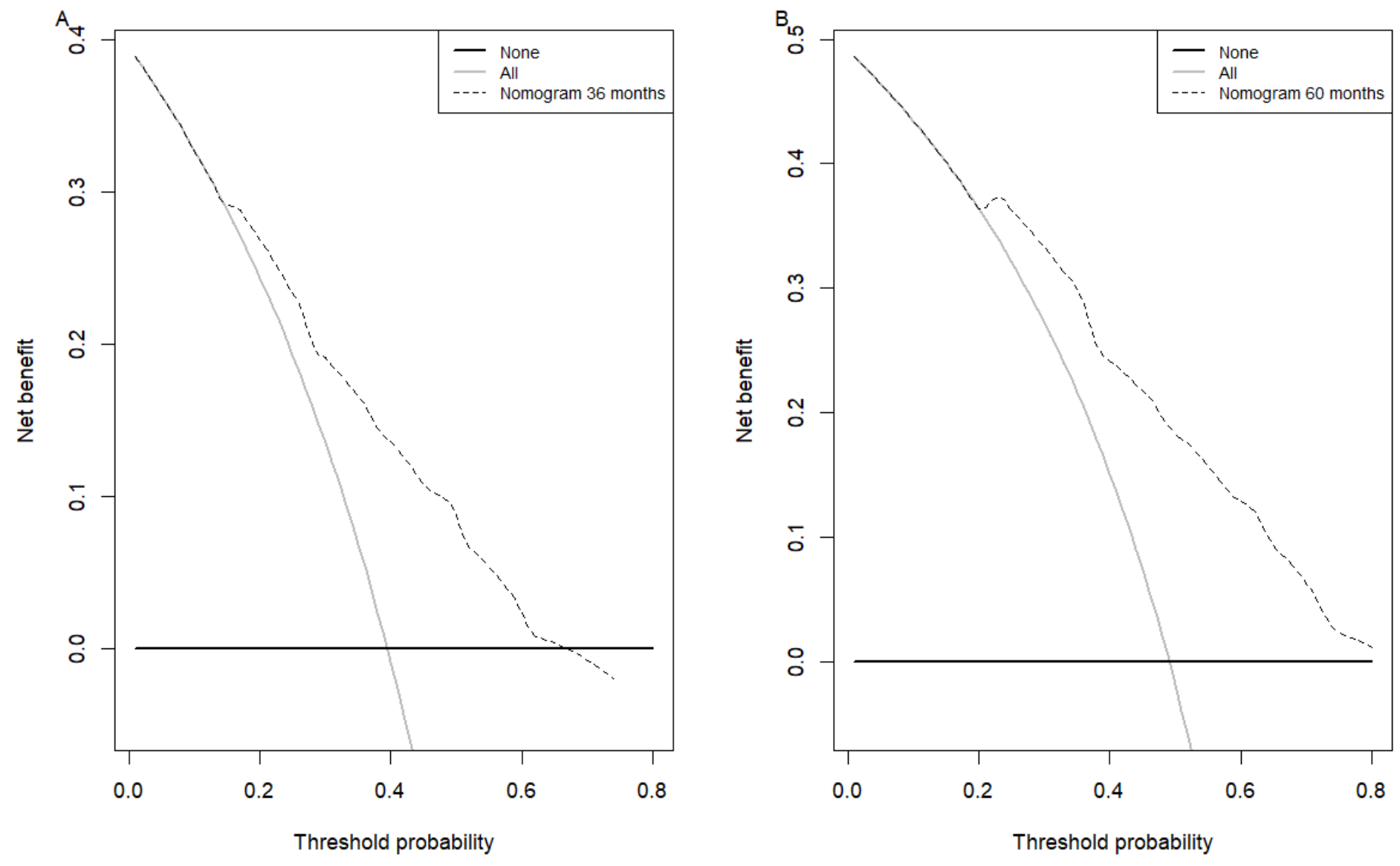

Figure 8

DCA to evaluate clinical utility of nomogram. 